\title{
Josephson oscillations of chirality and identity in two-dimensional solitons in spin-orbit-coupled condensates
}

\author{
Zhaopin Chen $\odot,{ }^{1}$ Yongyao $\mathrm{Li},{ }^{2,1}$ and Boris A. Malomed $\odot^{1,3}$ \\ ${ }^{1}$ Department of Physical Electronics, School of Electrical Engineering, Faculty of Engineering, Tel Aviv University, Tel Aviv 69978, Israel \\ ${ }^{2}$ School of Physics and Optoelectronic Engineering, Foshan University, Foshan 52800, China \\ ${ }^{3}$ Instituto de Alta Investigación, Universidad de Tarapacá, Casilla 7D, Arica, Chile
}

(Received 10 February 2020; revised 3 April 2020; accepted 26 July 2020; published 7 August 2020)

\begin{abstract}
We investigate dynamics of 2D chiral solitons of semivortex (SV) and mixed-mode (MM) types in spinorbit-coupled Bose-Einstein condensates with the Manakov nonlinearity, loaded in a dual-core (double-layer) trap. The system supports two novel manifestations of Josephson phenomenology: one in the form of persistent oscillations between SVs or MMs with opposite chiralities in the two cores, and another one demonstrating robust periodic switching (identity oscillations) between SV in one core and MM in the other, provided that the strength of the intercore coupling exceeds a threshold value. Below the threshold, the system either creates composite states, which are asymmetric with respect to the two cores, or collapses. Robustness of the chirality and identity oscillations against deviations from the Manakov nonlinearity is investigated too. These dynamical regimes are possible only in the nonlinear system. In the linear one, exact stationary and dynamical solutions for SVs and MMs of the Bessel type are found. They sustain Josephson self-oscillations in different modes, with no interconversion between them.
\end{abstract}

DOI: 10.1103/PhysRevResearch.2.033214

\section{INTRODUCTION}

Josephson oscillations, induced by tunneling of wave functions between weakly coupled cores, is a ubiquitous effect in macroscopic quantum systems [1]. It has been predicted and observed in superconductors separated by a thin insulating layer [2-12], superfluid ${ }^{3} \mathrm{He}$ [13], atomic Bose-Einstein condensates (BECs) [14-21], optical couplers [22-25], and exciton-polariton waveguides [26]. Among other applications, the Josephson effect in superconductors may be used for design of qubits $[27,28]$. Josephson oscillations of angular momentum between annular BECs was investigated too [29-38]. In these contexts, chirality of the wave functions may play an important role [29], [39-44].

In its linear form, the Josephson effect only gives rise to plasma waves in superconducting junctions. The most significant modes in the junctions are topological solitons (fluxons) supported by the nonlinear Josephson relation between the tunneling current and phase difference across the junction. Both classical [7-10] and quantum [11] fluxons, as well as superfluxons, i.e., kink excitations in fluxon chains [12], were studied theoretically and experimentally.

New possibilities for realization of dynamical effects in BEC are offered by binary (pseudo-spinor) condensates with spin-orbit coupling (SOC) between their components [45-48]. In particular, SOC affects the Josephson tunneling between

Published by the American Physical Society under the terms of the Creative Commons Attribution 4.0 International license. Further distribution of this work must maintain attribution to the author(s) and the published article's title, journal citation, and DOI. annular condensates [49]. Stable 2D solitons, which include vortex components, were predicted in the SOC system of the Rashba type [50] with attractive nonlinear interactions. In terms of optics, these interactions are represented by self- and cross-phase-modulation (SPM and XPM) terms in the underlying system of coupled Gross-Pitaevskii equations. While in the absence of SOC, attractive cubic terms in 2D equations always lead to collapse [51,52] (i.e., the system does not have a ground state), the interplay of the attractive SPM and XPM terms with SOC gives rise to ground states, in the form of solitons of the semivortex (SV, alias half-vortex [53,54]) or mixed-mode (MM) types, provided that, respectively, the SPM nonlinearity is stronger or weaker than its XPM counterpart. SVs are composed of a vortex in one component and zerovorticity soliton in the other, while MMs combine vortical and zero-vorticity terms in both components. In the experiment, such states can be created by using helical laser beams that resonantly couple to one component only, and thus transfer the angular momentum onto that component, without exciting the other one [55-57]. Two or several 2D solitons in the system's plane can be created too, by means of an initially applied cellular in-plane potential, which cuts the condensate into lateral fragments, and is lifted afterwards [58].

If the SV soliton is the ground state, the MM is an unstable excited one, and vice versa. Similar results were reported [59] for more general SOC systems, of the Rashba-Dresselhaus [60] type. Two-dimensional SV solitons exist in the form of two different chiral isomers (right- and left-handed ones), with vorticity sets in their components being, respectively, $\left(S_{+}, S_{-}\right)=(0,1)$ or $(-1,0)$ (mirror images of each other). Similarly, there exist two different chiral forms of MMs, which are introduced below in Eq. (22). 
In the experimental realization of SOC in binary BEC, two components are represented by different hyperfine states of the same atom, with nearly equal strengths of the SPM and XPM interactions, suggesting one to consider the Manakov nonlinearity [61], with equal SPM and XPM coefficients. In such a case, the system is invariant with respect to rotation of the pseudo-spinor wave function in the plane of its two components. The 2D system combining SOC and the Manakov nonlinearity gives rise to an additional soliton family, which embeds the SV and MM solitons into a continuous set of intermediate states [53]. The family is a degenerate one, in the sense that all solitons with a fixed total norm share common values of the energy and chemical potential. The family is dynamically stable against small perturbations, but structurally unstable, as a deviation from the SPM = XPM condition breaks the intermediate states, keeping only the SV and MM solitons as robust modes.

The subject of this work is a junction formed by a pair of Josephson-coupled 2D layers (cores), each one carrying spin-orbit-coupled BEC. In the experiment, a two-layer setup can be realized by loading the condensate in two adjacent valleys of a deep $1 \mathrm{D}$ optical lattice, which illuminates the setting in the perpendicular direction (see, e.g., Ref. [62]). In a general form, a dual-core 2D BEC system was introduced in Ref. [63]. In that work, 2D solitons, built of components in the two layers, were stabilized by a spatially periodic in-plane potential, while SOC was not considered. The problem addressed in Ref. [63] was spontaneous breaking of the intercore symmetry in the solitons, but not Josephson oscillations. Here, we consider oscillations between Josephson-coupled 2D SOC condensates, of both SV and MM types, in the dual-core system. In its linear version, Bessel-shaped exact solutions are found in an analytical form, for stationary states and Josephson oscillatory ones alike. These solutions demonstrate solely intrinsic oscillations in the two components of the condensate, which do not mix states of the SV and MM types, nor different chiral isomers in each type. In the full nonlinear system, solutions for self-trapped states are obtained in a numerical form. They readily demonstrate robust periodic oscillations between components of the $2 \mathrm{D}$ solitons with opposite chiralities in the two layers. Furthermore, Josephsontype identity oscillations, i.e., periodic mutual interconversion between solitons' components of the SV and MM types in the two layers, are reported too.

The rest of the paper is structured as follows. The model is formulated in Sec. II, in which basic types of the considered states are introduced too, including new solutions for the Bessel-shaped SV and MM modes in the linear system. Results for the Josephson oscillations are collected in Sec. III. It includes exact solutions obtained in the linear system, and a summary of the systematic numerical analysis of Josephson oscillations in 2D solitons of different types in the full nonlinear system. The numerical results make it possible to identify robust regimes of oscillations of the chirality (right $\rightleftarrows$ left) and identity (SV $\rightleftarrows \mathrm{MM}$ ) between the coupled layers. The paper is summarized in Sec. IV, where we also give estimates of the predicted effects in physical units, and discuss directions for the further work; one of them may be the use of beyond-mean-field effects [51] for the stabilization of the
2D system against the critical collapse [52], when its norm exceeds the respective threshold value.

\section{THE MODEL: COUPLED GROSS-PITAEVSKII EQUATIONS}

\section{A. The single-layer spin-orbit-coupled (SOC) system}

For the single effectively two-dimensional layer, the system of coupled Gross-Pitaevskii equations for components $\phi_{ \pm}$ of the pseudospinor wave function, coupled by the spin-orbit interaction of the Rashba type, is written as $[53,59]$

$$
\begin{aligned}
& i \partial_{t} \phi_{+}=-\left[\frac{1}{2} \nabla^{2}+\left(\left|\phi_{+}\right|^{2}+\gamma\left|\phi_{-}\right|^{2}\right)\right] \phi_{+}+\left(\partial_{x}-i \partial_{y}\right) \phi_{-}, \\
& i \partial_{t} \phi_{-}=-\left[\frac{1}{2} \nabla^{2}+\left(\left|\phi_{-}\right|^{2}+\gamma\left|\phi_{+}\right|^{2}\right)\right] \phi_{-}-\left(\partial_{x}+i \partial_{y}\right) \phi_{+},
\end{aligned}
$$

where $t$ and $x, y$ are scaled time and coordinates, the SPM and SOC coefficients (the latter one is the coefficient in front of the first-order spatial derivatives) are set to be 1 by scaling, and $\gamma$ is the relative XPM strength. We will chiefly address the Manakov nonlinearity, with $\gamma=1$, which is close to the experimentally relevant situation [45-48]; effects of deviation from the Manakov's case are considered too. Equations (1) and (2) do not include an external trapping potential, which is necessarily present in the experiment, but its effect on stable solitons is usually negligible [53].

Stationary self-trapped states of the pseudo-spinor condensate (solitons) with chemical potential $\mu<0$ are looked for as

$$
\phi_{ \pm}(x, y, t)=\Phi_{ \pm}(x, y) e^{-i \mu t},
$$

with the $2 \mathrm{D}$ norm

$$
N_{\phi}=N_{+}+N_{-} \equiv \iint\left[\left|\Phi_{+}(x, y)\right|^{2}+\left|\Phi_{-}(x, y)\right|^{2}\right] d x d y .
$$

Two isomers of solitons of the SV type, with right- and left-handed chiralities, are defined, respectively, by vorticity sets $\left(S_{+}, S_{-}\right)=(0,+1)$ and $\left(\bar{S}_{+}, \bar{S}_{-}\right)=(-1,0)$ in the two components. Numerical solutions for them are produced, severally, by the following inputs:

$$
\begin{gathered}
\phi_{+}^{(0)}=A_{1} \exp \left(-\alpha_{1} r^{2}\right), \phi_{-}^{(0)}=A_{2} r \exp \left(i \theta-\alpha_{2} r^{2}\right), \\
\overline{\phi_{+}^{(0)}}=-A_{2} r \exp \left(-i \theta-\alpha_{2} r^{2}\right), \overline{\phi_{-}^{(0)}}=A_{1} \exp \left(-\alpha_{1} r^{2}\right),
\end{gathered}
$$

which are written in polar coordinates $(r, \theta)$, with real $A_{1,2}$ and $\alpha_{1,2}>0$. These inputs are natural, as Eqs. (1) and (2) are fully compatible with the substitution of SV Ansätze with opposite chiralities [53],

$$
\begin{gathered}
\left\{\phi_{+}, \phi_{-}\right\}_{\mathrm{SV}}=e^{-i \mu t}\left\{f(r), e^{i \theta} g(r)\right\}, \\
\left\{\overline{\phi_{+}}, \overline{\phi_{-}}\right\}_{\mathrm{SV}}=e^{-i \mu t}\left\{-e^{-i \theta} g(r), f(r)\right\},
\end{gathered}
$$

where radial form-factors $f(r), g(r)$ obey equations

$$
\begin{gathered}
{\left[\mu+\frac{1}{2}\left(\frac{d^{2}}{d r^{2}}+\frac{1}{r} \frac{d}{d r}\right)+2\left(f^{2}+g^{2}\right)\right] f} \\
-\left(\frac{1}{r} g+\frac{d g}{d r}\right)=0,
\end{gathered}
$$




$$
\begin{gathered}
{\left[\mu+\frac{1}{2}\left(\frac{d^{2}}{d r^{2}}+\frac{1}{r} \frac{d}{d r}\right)+2\left(f^{2}+g^{2}\right)\right] g} \\
-\left(\frac{1}{2 r^{2}} g-\frac{d f}{d r}\right)=0 .
\end{gathered}
$$

More general soliton modes are introduced below.

\section{B. Exact Bessel modes of the linearized single-layer system}

Equations (9) and (10) give rise to solitons if $\mu$ belongs to the respective semi-infinite band gap, $\mu<-1 / 2$ [see Eq. (33) below, with $\kappa=0$ ]. At $r \rightarrow \infty$, the linearization of Eqs. (9) and (10) readily predicts that exponentially decaying tails of solitons' form-factors $f(g)$ and $g(r)$ are built as combinations of terms

$$
(f, g)_{\text {tail }} \sim r^{-1 / 2} \exp (-\sqrt{-(2 \mu+1)} r)\{\cos r, \sin r\} .
$$

Furthermore, the linearized equations make it possible to find exact solutions for weakly localized Bessel-shaped modes (with a diverging total norm) in the propagation band (at $\mu>$ $-1 / 2$ ), which resemble exact nondiffracting states in linear optics, cf. Refs. [64-66]). At $\mu>0$, the solutions of the SV type are

$$
f_{\text {lin }}(r)=A^{(0)} J_{0}(r / \rho), g_{\text {lin }}(r)=A^{(0)} s J_{1}(r / \rho)
$$

with arbitrary amplitude $A^{(0)}$, Bessel functions $J_{0,1}$, sign factor $s= \pm 1$ (this sign is independent from subscripts in $\phi_{ \pm}, \psi_{ \pm}$), and

$$
\rho=\rho_{s} \equiv(\sqrt{1+2 \mu}+s) /(2 \mu) .
$$

In the remaining part of the propagation band, $-1 / 2<\mu<0$, solution (12) is relevant only for $s=-1$, while expression (13) is replaced by

$$
\rho=\rho_{ \pm} \equiv(1 \pm \sqrt{1+2 \mu}) /(-2 \mu),
$$

with another independent sign \pm .

At the edge of the semi-infinite gap, $\mu=-1 / 2$, Eq. (14) gives a single solution with $\rho=1$,

$$
\begin{aligned}
& f_{\text {lin }}^{(1)}\left(r ; \mu=-\frac{1}{2}\right)=A^{(0)} J_{0}(r), \\
& g_{\operatorname{lin}}^{(1)}\left(r ; \mu=-\frac{1}{2}\right)=-A^{(0)} J_{1}(r),
\end{aligned}
$$

instead of two, produced inside the gap at $\mu>-1 / 2$. However, the loss of one solution is compensated by the existence of an additional one, precisely at the edge of the gap. Its components are

$$
\begin{aligned}
f_{\text {lin }}^{(2)}\left(r ; \mu=-\frac{1}{2}\right) & \equiv \lim _{\mu+1 / 2 \rightarrow+0} \frac{f_{\text {lin }}\left(r ; \rho_{+}\right)-f_{\text {lin }}\left(r ; \rho_{-}\right)}{2 \sqrt{1+2 \mu}} \\
& =-A^{(0)} r J_{1}(r), \\
g_{\text {lin }}^{(2)}\left(r ; \mu=-\frac{1}{2}\right) & \equiv \lim _{\mu+1 / 2 \rightarrow+0} \frac{g_{\operatorname{lin}}\left(r ; \rho_{+}\right)-g_{\operatorname{lin}}\left(r ; \rho_{-}\right)}{2 \sqrt{1+2 \mu}} \\
& =A^{(0)}\left[r J_{2}(r)-J_{1}(r)\right] .
\end{aligned}
$$

Exact linear SV states with the opposite chirality are generated from expressions (12)-(16) as per Eq. (8). Next, MMs states, as exact solutions of the linear SOC system, are constructed from the SVs pursuant to Eqs. (22) and (23). It is also relevant to mention that, for the Rashba SOC replaced by the Dresselhaus form [60], the exact linear solutions for the SV and MM modes are essentially the same as found here for the Rashba system.

In addition to solution (12), which represents the basic SV state in the linear system, it is possible to construct exact solutions for excited states, obtained by injection of extra integer vorticity $\Delta S=1,2, \ldots$ in both components of the SV state. Such solutions are obtained replacing Eqs. (12), (15), and (16), severally, by

$$
\begin{aligned}
& f_{\operatorname{lin}}(r)= A^{(0)} J_{\Delta S}(r / \rho), g_{\operatorname{lin}}(r)=A^{(0)} J_{1+\Delta S}(r / \rho) \\
& f_{\text {lin }}^{(1)}\left(r ; \mu=-\frac{1}{2}\right)=A^{(0)} J_{\Delta S}(r) \\
& g_{\text {lin }}^{(1)}\left(r ; \mu=-\frac{1}{2}\right)=-A^{(0)} J_{1+\Delta S}(r), \\
& f_{\text {lin }}^{(2)}\left(r ; \mu=-\frac{1}{2}\right)=A^{(0)}\left[\Delta S \cdot J_{\Delta S}(r)-r J_{1+\Delta S}(r)\right] \\
& g_{\operatorname{lin}}^{(2)}\left(r ; \mu=-\frac{1}{2}\right)=A^{(0)}\left[r J_{2+\Delta S}(r)-(1+\Delta S) J_{1+\Delta S}(r)\right],
\end{aligned}
$$

while Eqs. (13) and (14) keep the same form as above, as well as the possibility to construct the SV with the opposite chirality and MMs, according to Eqs. (8), (22), and (23).

It is relevant to mention that similarly defined excited states of SV and MM solitons are unstable in the nonlinear system, unlike the ground states with $\Delta S=0$ [53]. On the other hand, the excited-state solitons may be made stable in a model with self-trapping provided by the local repulsive nonlinearity growing fast enough from the center to periphery [67].

\section{Soliton families}

The Manakov's nonlinearity makes it possible to construct a general family of solitons, including SVs, MMs, and intermediate states. The creation of such states is initiated by a combination of inputs (5) and (6) with the opposite chiralities,

$$
\phi_{ \pm}=M \phi_{ \pm}^{(0)}+\sqrt{1-M^{2}} \cdot \overline{\phi_{ \pm}^{(0)}},
$$

where the weight factor takes values $-1 \leqslant M \leqslant 1$. Because integrals of cross-products, generated by Ansätze (5) and (6) vanish,

$$
\iint\left(\phi_{+}^{(0)}\right)^{*} \phi_{-}^{(0)} d x d y=0,
$$

the norm of input (20), defined as per Eq. (4), does not depend on $M$, hence this input gives rise to a family of solitons with the same norm.

The choice of $M=+1 / \sqrt{2}$ or $-1 / \sqrt{2}$ in Eq. (20) corresponds to MMs with opposite chiralities. Stationary MM solitons of the Manakov system are produced by the following exact Ansatz, which is compatible with the underlying system of equations (1) and (2) [cf. Eq. (7), that produces the exact Ansatz for SV modes]:

$$
\begin{aligned}
\left\{\phi_{+}, \phi_{-}\right\}_{\mathrm{MM}} & =\frac{1}{\sqrt{2}} e^{-i \mu t}\left\{f(r)-e^{-i \theta} g(r), f(r)+e^{i \theta} g(r)\right\} \\
& \equiv \frac{1}{\sqrt{2}}\left(\left\{\phi_{+}, \phi_{-}\right\}_{\mathrm{SV}}+\left\{\overline{\phi_{+}}, \overline{\phi_{-}}\right\}_{\mathrm{SV}}\right)
\end{aligned}
$$




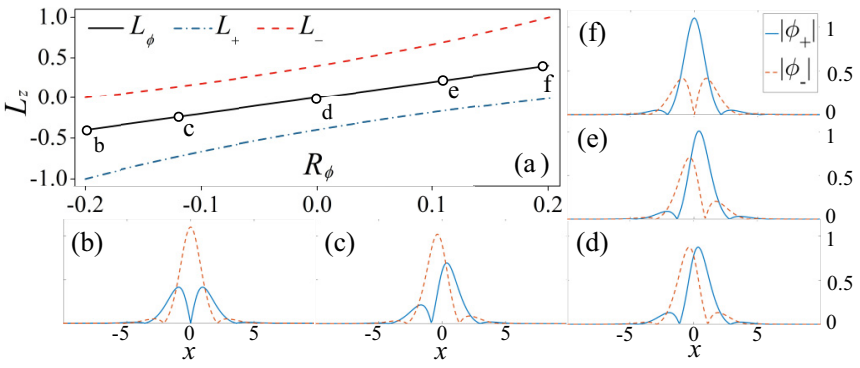

FIG. 1. (a) Angular momenta per particle $\left(L_{z}=L_{\phi}\right.$ or $L_{ \pm}$, as indicated in the panel), which are defined by Eqs. (25) and (26), are shown as functions of norm ratio $R_{\phi}$ [see Eq. (24)], for the continuous family of stable SOC solitons, numerically generated by input (20), with fixed norm $N_{\phi}=4$, in the single-core system. Linear dependence $L_{\phi}\left(R_{\phi}\right)$ follows Eq. (27). The energy and chemical potential of all solutions belonging to the degenerate family are $E_{\phi}=-2.4$ and $\mu=-0.87$. Cross-sections of two components of the 2D solitons, marked in (a), are displayed for the following values of integral quantities (24) and (25), (26): $R_{\phi}=-0.2, L_{\phi}=-0.4$ (b); $R_{\phi}=-0.12, L_{\phi}=-0.24$ (c); $R_{\phi}=0, L_{\phi}=0$ (d); $R_{\phi}=0.11$, $L_{\phi}=0.22$ (e); and $R_{\phi}=0.2, L_{\phi}=0.4$ (f).

Here, $f$ and $g$ are the same real functions which are introduced above as solutions to Eqs. (9) and (10). Another chiral MM isomer is produced by a different Ansatz,

$$
\begin{aligned}
\left\{\overline{\phi_{+}}, \overline{\phi_{-}}\right\}_{\mathrm{MM}} & =\frac{1}{\sqrt{2}} e^{-i \mu t}\left\{f(r)+e^{-i \theta} g(r),-f(r)+e^{i \theta} g(r)\right\} \\
& \equiv \frac{1}{\sqrt{2}}\left(\left\{\phi_{+}, \phi_{-}\right\}_{\mathrm{SV}}-\left\{\overline{\phi_{+}}, \overline{\phi_{-}}\right\}_{\mathrm{SV}}\right)
\end{aligned}
$$

with radial form-factors $f$ and $g$ obeying the same equations (9) and (10). The exact linear relations between the MM and SVs in Eqs. (22) and (23) are admitted by the symmetry of the Manakov nonlinearity. These relations entail an equality for the total norms (4): $N_{\mathrm{MM}}(\mu)=N_{\mathrm{SV}}(\mu)$. To the best of our knowledge, the exact MM Ansätze represented by Eqs. (22) and (23) were not reported earlier.

The solitons are characterized by the norm ratio between the components,

$$
R_{\phi}=\left(N_{\phi_{+}}-N_{\phi_{-}}\right) /\left(N_{\phi_{+}}+N_{\phi_{-}}\right),
$$

and by the angular momentum per particle in each component and in the entire system,

$$
\begin{gathered}
L_{ \pm}=N_{ \pm}^{-1} \iint \phi_{ \pm}^{*} \hat{\mathcal{L}}_{z} \phi_{ \pm} d x d y \\
L_{\phi}=N_{\phi}^{-1} \sum_{+,-} \iint \phi_{ \pm}^{*} \hat{\mathcal{L}}_{z} \phi_{ \pm} d x d y,
\end{gathered}
$$

where the angular-momentum operator is $\hat{\mathcal{L}}_{z}=-i(x \partial / \partial y-$ $y \partial / \partial x) \equiv-i \partial / \partial \theta$, and the norms are taken as per Eq. (4). For the family generated by Ansatz (20), a typical relation between $L$ and $R_{\phi}$ is displayed in Fig. 1(a). The linear $L_{\phi}\left(R_{\phi}\right)$ dependence, observed in the figure, is explained by the fact that the input in the form of Egs. (5)-(20), leads, taking into regard the orthogonality of $\phi_{+}^{(0)}$ and $\phi_{-}^{(0)}$ [see Eq. (21)], to relations $R_{\phi}(M)=\left(2 M^{2}-1\right) R_{\phi}^{(\mathrm{SV})}$ and

$$
L_{\phi}\left(R_{\phi}\right)=\left(1-R_{\phi}^{(\mathrm{SV})}\right)\left(2 R_{\phi}^{(\mathrm{SV})}\right)^{-1} R_{\phi},
$$

where $R_{\phi}^{(\mathrm{SV})}$ is given by Eq. (24) for the SV soliton corresponding to $M=1$ in Eq. (20)) [in Fig. 1(a), a numerically computed value is $R_{\phi}^{(\mathrm{SV})}=0.2$ ].

Both $R_{\phi}$ and $L_{\phi}$ vanish for solitons of the MM type, due to the overall symmetry between the components in this state. Terminal points of the curves in Fig. 1(a) correspond to $|M|=$ 1 or $M=0$ in Eq. (20), i.e., SV solitons, in which either $L_{+}= \pm 1$ and $L_{-}=0$, or vice versa. Numerical data yield $L_{\phi}= \pm 0.4$ in Eq. (27) at the terminal points. In other states, which correspond to $0<|M|<1$ in Eq. (20), the numerical results give $-0.4<L_{\phi}<0.4$. Examples of solitons belonging to the family are presented in Figs. 1(b)-1(f). In particular, SVs are shown in panels (b) and (f), while (d) displays an MM. The stability of the entire family was established in direct simulations. The solitons of all types exist in ranges $0<-\mu<\infty$ and $0<N_{\phi}<N_{\text {coll }} \approx 5.85$, where $N_{\text {coll }}$ is the well-known threshold for the onset of the critical collapse, predicted by the single Gross-Pitaevskii equation [52]. In fact, the stability of the 2D spin-orbit-coupled solitons is predicated on the fact that their total norm takes values $N<N_{\text {coll }}$, thus securing them against the onset of the collapse [53].

\section{The Josephson-coupled dual-core system}

The pair of Josephson-coupled layers, each carrying pseudo-spinor BEC, are modeled by the system of equations for four components of wave functions, $\phi_{ \pm}$in one layer and $\psi_{ \pm}$in the other:

$$
\begin{aligned}
i \partial_{t} \phi_{+}= & -\left[\frac{1}{2} \nabla^{2}+\left(\left|\phi_{+}\right|^{2}+\gamma\left|\phi_{-}\right|^{2}\right)\right] \phi_{+} \\
& +\left(\partial_{x}-i \partial_{y}\right) \phi_{-}-\kappa \psi_{+}, \\
i \partial_{t} \phi_{-}= & -\left[\frac{1}{2} \nabla^{2}+\left(\left|\phi_{-}\right|^{2}+\gamma\left|\phi_{+}\right|^{2}\right)\right] \phi_{-} \\
& -\left(\partial_{x}+i \partial_{y}\right) \phi_{+}-\kappa \psi_{-}, \\
i \partial_{t} \psi_{+}= & -\left[\frac{1}{2} \nabla^{2}+\left(\left|\psi_{+}\right|^{2}+\gamma\left|\psi_{-}\right|^{2}\right)\right] \psi_{+} \\
& +\left(\partial_{x}-i \partial_{y}\right) \psi_{-}-\kappa \phi_{+}, \\
i \partial_{t} \psi_{-}= & -\left[\frac{1}{2} \nabla^{2}+\left(\left|\psi_{-}\right|^{2}+\gamma\left|\psi_{+}\right|^{2}\right)\right] \psi_{-} \\
& -\left(\partial_{x}+i \partial_{y}\right) \psi_{+}-\kappa \phi_{-},
\end{aligned}
$$

cf. Eqs. (1) and (2), where $\kappa$ is the Josephson-coupling strength, which is defined to be positive. Note that the Josephson interaction does not linearly couple components $\phi_{ \pm}$to $\psi_{\mp}$, as ones with opposite subscripts represent two distinct atomic states, and the tunneling between the parallel layers does not include a mechanism which would lead to mutual transformation of different atomic states.

The system of Eqs. (28)-(31) conserves the total norm, $N_{\phi}+N_{\psi}$, where $N_{\psi}$ is a counterpart of the $\phi$ norm for the $\psi$ layer, see Eq. (4). Other dynamical invariants of the system 
are the total energy,

$$
\begin{aligned}
E= & \iint\left\{\sum _ { \chi = \phi , \psi } \left[\frac{1}{2}\left(\left|\nabla \chi_{+}\right|^{2}+\left|\nabla \chi_{-}\right|^{2}-\left|\chi_{+}\right|^{4}-\left|\chi_{-}\right|^{4}\right)\right.\right. \\
& \left.-\gamma\left|\chi_{+} \chi_{-}\right|^{2}+\left(\left(\chi_{+}^{*} \partial_{x} \chi_{-}-i \chi_{+}^{*} \partial_{y} \chi_{-}\right)+\text {c.c. }\right)\right] \\
& \left.-\kappa\left(\left(\phi_{+}^{*} \psi_{+}+\phi_{-}^{*} \psi_{-}\right)+\text {c.c. }\right)\right\} d x d y,
\end{aligned}
$$

and the total angular momentum, $N_{\phi} L_{\phi}+N_{\psi} L_{\psi}$, cf. Eq. (26).

\section{ANALYTICAL AND NUMERICAL RESULTS FOR JOSEPHSON OSCILLATIONS}

A. Exact solutions for Josephson oscillations in the linear system

Linearization of Eqs. (28)-(31) for excitations $\left(\phi_{ \pm}, \psi_{ \pm}\right) \sim$ $\exp \left(i q_{x} x+i q_{y} y-i \mu t\right)$ yields four branches of the dispersion relation:

$$
\mu=\frac{1}{2}\left(q_{x}^{2}+q_{y}^{2}\right)+\sigma_{1} \sqrt{q_{x}^{2}+q_{y}^{2}}-\sigma_{2} \kappa,
$$

with two mutually independent sign parameters $\sigma_{1,2}= \pm 1$ (which are unrelated to subscripts of $\phi_{ \pm}$and $\psi_{ \pm}$). Solitons may exist in the semi-infinite gap, readily determined as an interval of values of $\mu$ which is not covered by Eq. (33) with $-\infty<q_{x, y}<+\infty$ and all choices of $\sigma_{1,2}$ :

$$
\mu_{\text {sol }}<-(1 / 2+\kappa)
$$

(remind we fix $\kappa>0$, by definition). In particular, this gap is obtained from the above-mentioned one, $\mu_{\text {sol }}<-1 / 2$, for the single-layer system, if one considers symmetric solutions of Eqs. (28)-(31), with $\psi_{ \pm}=\phi_{ \pm}$. On the other hand, for antisymmetric solutions, with $\psi_{ \pm}=-\phi_{ \pm}$, the single-layer gap is replaced by an expanded one, $\mu_{\text {sol }}<-(1 / 2-\kappa)$. In the additional interval which does not belong to gap (34), viz., $-(1 / 2+\kappa)<\mu_{\text {sol }}<-(1 / 2-\kappa)$, antisymmetric states may exist as embedded solitons [68], but they are unstable.

Further, the linearized system gives rise to exact solutions for Josephson-oscillation states,

$$
\begin{aligned}
\left(\phi_{ \pm}(x, y, t)\right)_{\text {two-layer }} & =\cos (\kappa t) \cdot\left(\phi_{ \pm}(x, y, t)\right)_{\text {single-layer }}, \\
\left(\psi_{ \pm}(x, y, t)\right)_{\text {two-layer }} & =i \sin (\kappa t) \cdot\left(\phi_{ \pm}(x, y, t)\right)_{\text {single-layer }},
\end{aligned}
$$

where $\left(\phi_{ \pm}(x, y, t)\right)_{\text {single-layer }}$ are the component of any solution of the linearized version of Eqs. (1) and (2). In fact, explicit single-layer solutions which may be substituted in Eq. (35) are those given above by Eqs. (12)-(19). Note that the Josephson oscillations do not change the chemical potential in Eq. (35), which is borrowed from the respective single-layered solutions, that contain the factor of $\exp (-i \mu t)$.

\section{B. Josephson oscillations in 2D solitons}

\section{Chirality and identity oscillations}

In the framework of the full nonlinear system, we first address the evolution initiated by an input composed of a stable single-layer SV soliton, with vorticities $\left(S_{+}, S_{-}\right)=$

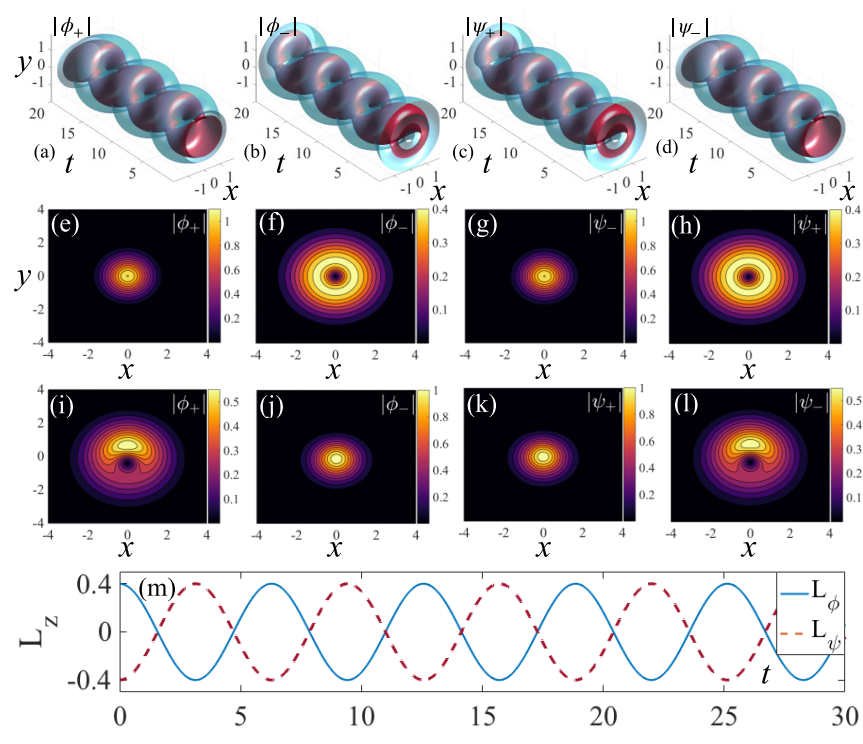

FIG. 2. Numerically simulated Josephson oscillations of chirality (with random-noise perturbation at the $1 \%$ amplitude level, added for testing stability of the dynamical regime), initiated by the input composed of a stable single-core SV in one layer, and its left-handed counterpart in the other, see Eqs. (7) and (8). Panels (a), (b) and (c), (d) display the evolution of the pseudo-spinor components in the first and second cores, respectively. The norm of the input in each layer is $N=4$ (hence the total norm in the two-layer system is 8), and the Josephson coupling constant is $\kappa=0.5$. (e)-(h) and (i)-(l) exhibit $2 \mathrm{D}$ density distributions of the components at, respectively, $t=0$ and $t=10$. (m) The respective oscillations of angular momenta per particle in both layers, $L_{\phi}$ and $L_{\psi}$, see Eq. (26).

$(0,1)$, in one core, and its chiral (left-handed) isomer with $\left(S_{+}, S_{-}\right)=(-1,0)$ in the other, which are created, at $t=0$, as per Ansätze presented in Eqs. (7) and ( 8). The respective stationary single-layer solutions were produced by means of the accelerated imaginary-time-evolution method [69], and their stability was then tested by simulations of Eqs. (1) and (2) in real time.

Simulations of Eqs. (28)-(31) with such an input produce a robust regime of periodic transmutations displayed in Figs. 2(a)-2(d). Each quarter period, SVs in both cores switch into their chiral counterparts, returning to the initial state, but with the opposite sign, each half period. Thus chiralities of the two SVs periodically flip, remaining opposite in the two cores, while the corresponding density patterns oscillate. Distributions of densities of the four involved components, displayed at $t=0$ in panels (e)-(f), and at $t=10$ in (i)-(l) (the latter time is close to 0.8 periods of the Josephson oscillations) help to understand the evolution of the density patterns in the course of the oscillations. Further, Fig. 2(m) demonstrates Josephson oscillations of the angular momentum in the same dynamical state, with the momentum per particle in the cores oscillating between $L_{\phi, \psi}= \pm 0.4$ and $\mp 0.4$.

Note that the chirality-switching Josephson-oscillation mode is possible only in the nonlinear system, as the exact linear Josephson-oscillation solution (35) admits solely oscillations that do not mix different chiralities. Indeed, the chirality flipping implies generation of new angular harmonics, which is not possible without nonlinearity. 

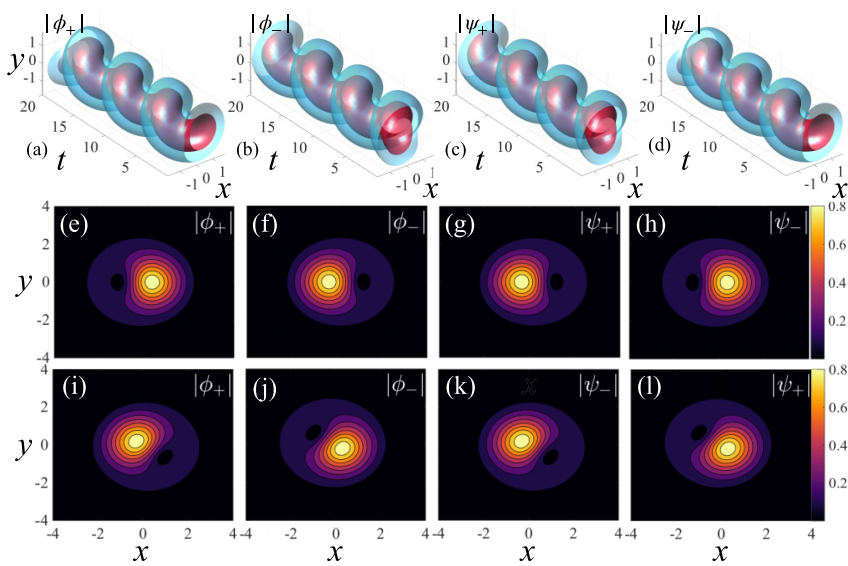

FIG. 3. The same as in Fig. 2 but for the input in the form of MMs with opposite chiralities in the two cores, and equal norms in them, $N=4$. The angular momentum per particle remains equal to zero in each core, due to the symmetry between the components of the pseudo-spinor wave function in the MM states. In addition to the Josephson oscillations of the MM chirality, the figure also demonstrates the spatiotemporal helicity in the oscillating soliton, see the text. Panels (e)-(h) and (i)-(1) exhibit 2D density distributions of the components at, respectively, $t=0$ and $t=10$.

Next, we consider Josephson oscillations between rightand left-handed chiral isomers of the MM type, which are created, at $t=0$, in the two layers, as per Ansätze (22) and (23). A typical numerical solution, displayed in Fig. 3, features stable periodic switching between the MM isomers in each layer. It also demonstrates spatiotemporal helicity, i.e., clockwise rotation of the density distributions (unlike nonrotating SV patterns in Fig. 2). The input with the opposite relative sign between the MM isomers in two layers gives rise to a similar stable dynamical state, but with rotation of the density profiles in the opposite, counter-clockwise, direction. Again, it is relevant to stress that flipping of the MM chirality in the course of the Josephson oscillations is only possible in the nonlinear system.

Recall that the single-core SOC system with the Manakov nonlinearity maintains the family of 2D solitons which are intermediate between the SV and MM states. Such states correspond to

$$
|M| \neq 0, \frac{1}{\sqrt{2}}, 1
$$

in Eq. (20), $\left|R_{\phi}\right| \neq 0.2$ or 0 in Fig. 1, and accordingly, eigenvalues $L_{\phi}$ different from \pm 0.4 and 0 , see Eq. (26). Systematic simulations of Josephson oscillations in pairs of chiral isomers of the intermediate states also reveal robust periodic chirality oscillations, in a combination with the rotation of the density profiles (the spatiotemporal helicity), similar to what is displayed above in Figs. 2 and 3 for the states of the SV and MM types. The results for the intermediate states are not displayed in a separate figure, as the overall pictures seem quite close to ones in Figs. 2 and 3. Furthermore, at all values of intercore coupling $\kappa$ the frequency of the oscillations and rotation is exactly equal to $\kappa$, cf. the exact solution of the linear system given by Eqs. (28)-(31). Although, as said
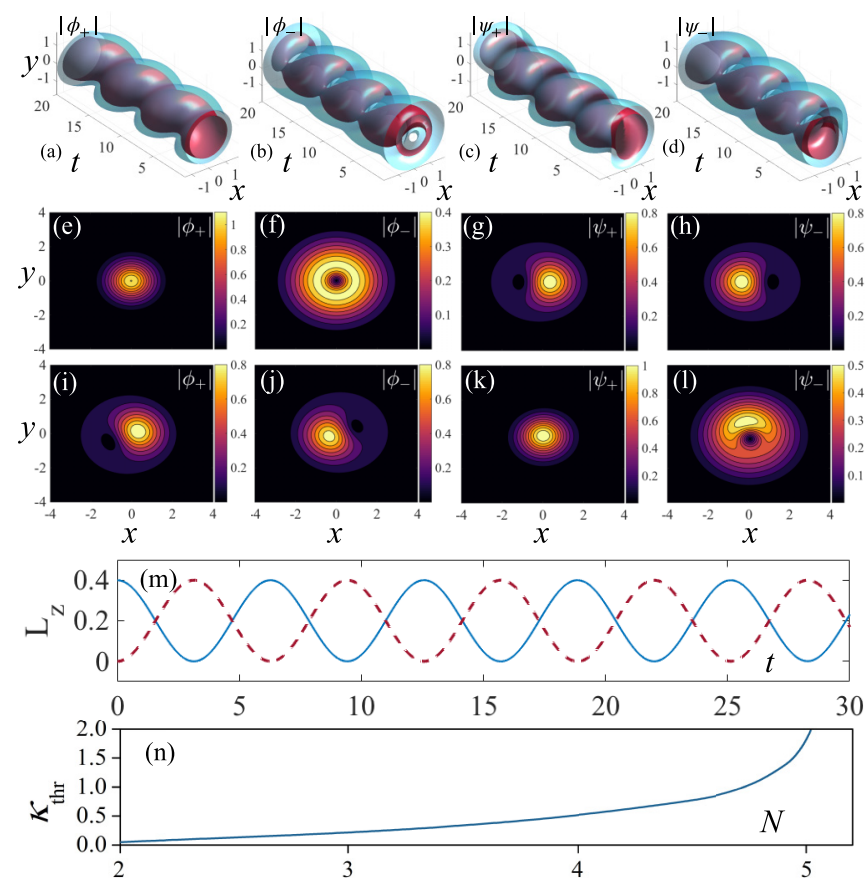

FIG. 4. [(a)-(m)] the same as in Fig. 2, but for identity oscillations, initiated by the input in the form of SV and MM solitons with equal norms, $N=4$, in the coupled cores. (n) The minimum (threshold) value of the intercore coupling $\kappa$, which is necessary for the stability of the identity oscillations, vs the norm of the 2D soliton in each core, $N$.

above, the chirality oscillations represent a nonlinear effect, the Josephson frequency is not affected by the nonlinearity, due to its "isotopic" invariance with respect to the rotation of the pseudo-spinor wave function in the plane of its two components.

The next step is to consider Josephson oscillations between inputs corresponding to SV in one core and $\mathrm{MM}$, with the same norm, in the other. In this case, the identity of the 2D solitons periodically switches between the SV and MM types in each core [see Figs. 4(a)-4(d) and 4(m)], therefore this stable dynamical state may be naturally categorized as identity oscillations, coupled to the clockwise helicity rotation, cf. Figs. 2 and 3. This regime, which is again possible solely in the nonlinear system, cf. exact solution (35) obtained in the linear limit (which actually corresponds to the system strongly dominated by the intercore coupling), takes place if the strength of the Josephson interaction exceeds a certain threshold value, $\kappa>\kappa_{\text {thr }}$. The dependence of the threshold on the total norm in one layer, $N$, is shown in Fig. 4(n); recall that the establishment of the robust chirality oscillations in the dual-core SV and MM solitons, considered above, does not require $\kappa$ to be larger than any finite threshold value.

To explain the latter feature, we note that, as it follows from Eqs. (28)-(32), the interplay of the kinetic energy, nonlinearity, SOC, and Josephson interaction between the cores predicts scaling $\kappa_{\text {thr }} \sim N$ at small $N$. On the other hand, the above-mentioned onset of the collapse in the single-core system at $N=N_{\text {coll }}$ implies divergence of the system's sensitivity to perturbations at $N \rightarrow N_{\text {coll }}$, hence $\kappa_{\text {thr }}$ also diverges in this 

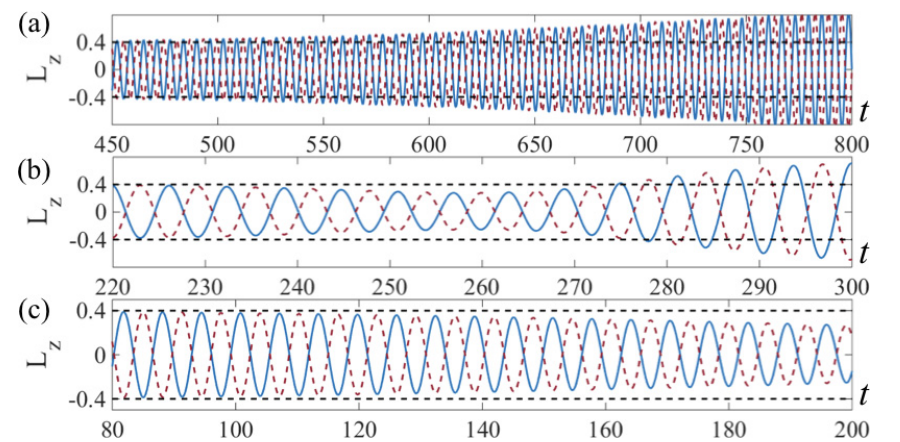

FIG. 5. Simulations akin to those displayed for the SV soliton in Fig. 2, but with $\gamma=1.05$ in (a) and 0.95 in (b). The chirality oscillations remain robust in the course of $\simeq 40$ and 20 oscillation periods, respectively. (c) The chirality oscillations of the SV solitons, with different initial norms in the two cores, $N_{\psi}=0.95 N_{\phi}=3.8$. The oscillations stay undisturbed in the course of $\simeq 10$ periods.

limit. These qualitative arguments are corroborated by the numerically found dependence $\kappa_{\mathrm{thr}}(N)$ in Fig. 4(n).

At $\kappa<\kappa_{\mathrm{thr}}$, the intercore coupling is too weak to prevent the occurrence of spontaneous symmetry breaking between the cores. Accordingly, additional numerical results demonstrate, for values of $N$ which are relatively small in comparison with $N_{\text {coll }}$ (namely, in an interval of $N<N_{\text {crit }}<$ $N_{\text {coll }}$ ) the existence of asymmetric composite solitons, with different amplitudes in the two cores. In the adjacent interval of $N_{\text {crit }}<N<N_{\text {coll }}$, the collapse takes place at $\kappa<\kappa_{\text {thr }}$. On the other hand, if the Josephson coupling is strong enough, with $\kappa>\kappa_{\text {thr }}$, it forestalls the onset of the collapse at all values of $N$ smaller than $N_{\text {coll }}$, by keeping the total norm equally distributed between the two cores (for instance, the identity-oscillation dynamical regime, displayed in Fig. 4, is stable at $N=4<N_{\text {coll }} \approx 5.85$ ).

\section{Structural stability of the oscillation regimes}

The fact that the Manakov's form of the nonlinearity is approximate in experimental settings makes it necessary to test effects of deviation from it $[\gamma \neq 1$ in Eqs. (1) and (2)], i.e., structural stability of the chirality- and identity-oscillation dynamical regimes. The result, illustrated by Figs. 5(a) and 5 (b) for $\gamma=1 \pm 0.05$, is that the chirality oscillations of the SV soliton persist in the course of dozens of periods (which is sufficient for the experimental observation), and then gradually detune from the regular regime.

Additional simulations (not shown here in detail) suggest similar conclusions concerning the effect of the deviation from the Manakov's case on chirality and identity oscillations of MM solitons. As concerns the intermediate states, defined above as per Eq. (36), the deviation from the Manakov's case for them is more disruptive, as, even in the single-core system, taking $\gamma \neq 1$ causes evolution of such inputs towards SVs or MMs, for $\gamma<1$ or $\gamma>1$, respectively [53].

Stability of the Josephson oscillations against variations of the initial conditions was tested too, by taking the input in the form of a single-core SV soliton in one layer, and its chiral isomer in the other, but with different norms. As shown in
Fig. 5(c), dephasing of the resulting chirality oscillations sets in at times exceeding $\sim 10$ periods.

\section{CONCLUSION}

The objective of this work is to introduce and theoretically elaborate a setting for the study of Josephson oscillations of the chirality and species type (identity) of 2D solitons in the double-layer trap, loaded with a spin-orbit-coupled condensate, including self- and cross-attractive nonlinearity (SPM and XPM) in its components. It is known that, in the Manakov's case of SPM = XPM, the single-layer system supports a broad family of composite 2D solitons, which includes dynamically and structurally stable ones of the SV (semi-vortex) and MM (mixed-mode) types, each existing in two chiral isomers, left- and right-handed ones. In addition to that, the Manakov's nonlinearity admits the existence of the intermediate family of solitons, between the SV and MM states, which are dynamically stable but structurally unstable against deviation from the condition SPM $=$ XPM. All these states exist with the norm in each component taking values smaller than the well-known threshold value necessary for the onset of the critical collapse, $N<N_{\text {coll }}$,

We consider Josephson oscillations initiated by inputs in the form of SV, MM, or intermediate-type solitons with equal norms and opposite chiralities, originally created in the coupled cores (layers), or SV in one core and MM in the other. In the former case, the system features robust chirality oscillations at all values of the intercore coupling, $\kappa$. In the latter case, persistent periodic switching between the SV and MM species (identity oscillations) occur at $\kappa>\kappa_{\mathrm{thr}}$, where $\kappa_{\mathrm{thr}}$ increases with the growth of the condensate's norm, while at $\kappa<\kappa_{\mathrm{thr}}$ the system develops states with unequal amplitudes in the cores at relatively small values of $N$, or collapses at larger $N$. In all cases, the oscillation frequency is equal to $\kappa$. Deviation of the nonlinearity from the Manakov's form, as well as a difference in the norms of the inputs in the two cores, leads to slowly developing dephasing of the Josephson oscillations. These regimes are nonlinear ones, while the exact solutions reported here for Josephson oscillations in the linear system admit solely self-oscillations of SVs and MMs modes with the Bessel spatial shape, without switching between different chiralities or mode types. In fact, the exact Bessel-shaped linear modes are new stationary solutions for the single-layer system too.

With typical values of the relevant physical parameters (see, e.g., Ref. [70]), such as the scattering length of the attractive interatomic interactions $\sim-0.1 \mathrm{~nm}$, transverse trapping frequency $\sim 300 \mathrm{~Hz}$, and the gap of width $\sim 5 \mu \mathrm{m}$ separating two layers, the expected number of atoms in the stable solitons, which are capable to feature the Josephson oscillations, is in the range of $N_{\text {atom }} \sim 10^{3}-10^{4}$, and the oscillation frequency $\sim 20 \mathrm{~Hz}$ (while the experiment time may extend up to $1 \mathrm{~s}$, making the observation of many cycles of the oscillations quite feasible). At essentially larger values of $N_{\text {atom }}$, the scaled norm will exceed value $N_{\text {coll }}$, which will trigger the onset of the collapse. On the other hand, it was recently predicted [51,71], and demonstrated experimentally in various setups [72-76], that 3D and quasi-2D self-trapped matterwave states may be stabilized, in the form of soliton-like 
quantum droplets, by beyond-mean-field (Lee-Huang-Yang) effects of quantum fluctuations around the mean-field states. In the present context, this possibility suggests to consider the dual-core SOC system in the case of $N>N_{\text {coll }}$, that may be stabilized by the Lee-Huang-Yang effect. In this connection, it is relevant to mention that the stabilization of $2 \mathrm{D}$ spin-orbitcoupled solitons by this effect in the single-layer system was analyzed in Ref. [77].

As another extension of the present work, it may be interesting to consider Josephson oscillations in 2D spatiotemporal optical solitons carried by dual-core planar waveguides and stabilized by a SOC-emulating mechanism, viz., temporal dispersion of the intercore coupling coefficient [78,79]. Another potentially interesting point is the study of intrinsic vibrations of the composite solitons, initiated by a separation of their components in the two layers.

\section{ACKNOWLEDGMENTS}

This work was supported, in part, by Grant No. 2019KZDXM001 from the Key Research Projects of General Colleges in Guangdong Province, and by Grant No. 11874112 from NNSFC (China), and by Grant No. 1286/17 from the Israel Science Foundation. Z.C. acknowledges an excellence scholarship provided by the Tel Aviv University.
[1] B. A. Malomed (ed.), Spontaneous Symmetry Breaking, SelfTrapping, and Josephson Oscillations (Springer, Berlin, 2013).

[2] B. D. Josephson, Possible new effects in superconductive tunneling, Phys. Lett. 1, 251 (1962).

[3] P. W. Anderson and J. M. Rowell, Probable Observation of the Josephson Superconducting Tunneling Effect, Phys. Rev. Lett. 10, 230 (1963).

[4] A. Barone and C. Paterno, Physics and Applications of the Josephson Effect (Wiley, New York, 1982).

[5] K. K. Likharev, Introduction to the Dynamics of Josephson Junctions (Nauka, Moscow, 1985) (in Russian).

[6] A. I. Buzdin, Proximity effects in superconductor-ferromagnet heterostructures, Rev. Mod. Phys. 77, 935 (2005).

[7] T. A. Fulton and R. C. Dynes, Single vortex propagation in Josephson tunnel junctions, Solid State Commun. 12, 57 (1973).

[8] A. V. Ustinov, M. Cirillo, and B. A. Malomed, Fluxon dynamics in one-dimensional Josephson-junction arrays, Phys. Rev. B 47, 8357(R) (1993).

[9] P. S. Lomdahl, Solitons in Josephson junctions - an overview, J. Stat. Phys. 39, 551 (1985).

[10] A. V. Ustinov, Solitons in Josephson junctions, Physica D 123, 315 (1998)

[11] A. Wallraff, A. Lukashenko, J. Lisenfeld, A. Kemp, M. V. Fistul, Y. Koval, and A. V. Ustinov, Quantum dynamics of a single vortex, Nature (London) 425, 155 (2003).

[12] B. A. Malomed, V. A. Oboznov, and V. A. Ustinov, Supersolitons in periodically inhomogeneous long Josephson junctions, Zh. Eksp. Teor. Fiz. 97, 924 (1990) [Sov. Phys. JETP 70, 518 (1990)].

[13] J. C. Davis and R. E. Packard, Superfluid ${ }^{3}$ He Josephson weak links, Rev. Mod. Phys. 74, 741 (2002).

[14] G. J. Milburn, J. Corney, E. M. Wright, and D. F. Walls, Quantum dynamics of an atomic Bose-Einstein condensate in a double-well potential, Phys. Rev. A 55, 4318 (1997).

[15] A. Smerzi, S. Fantoni, S. Giovanazzi, and S. R. Shenoy, Quantum Coherent Atomic Tunneling between Two Trapped BoseEinstein Condensates, Phys. Rev. Lett. 79, 4950 (1997).

[16] S. Raghavan, A. Smerzi, S. Fantoni, and S. R. Shenoy, Coherent oscillations between two weakly coupled Bose-Einstein condensates: Josephson effects, pi-oscillations, and macroscopic quantum self-trapping, Phys. Rev. A 59, 620 (1999).

[17] M. Albiez, R. Gati, J. Fölling, S. Hunsmann, M. Cristiani, and M. K. Oberthaler, Direct Observation of Tunneling and
Nonlinear Self-Trapping in a Single Bosonic Josephson Junction, Phys. Rev. Lett. 95, 010402 (2005)

[18] Y. Shin, G.-B. Jo, M. Saba, T. A. Pasquini, W. Ketterle, and D. E. Pritchard, Optical Weak Link between Two Spatially Separated Bose-Einstein Condensates, Phys. Rev. Lett. 95, 170402 (2005).

[19] S. Levy, E. Lahoud, I. Shomroni, and J. Steinhauer, The a.c. and d.c. Josephson effects in a Bose-Einstein condensate, Nature (London) 449, 579 (2007).

[20] G. Mazzarella, B. Malomed, L. Salasnich, M. Salerno, and F. Toigo, Rabi-Josephson oscillations and self-trapped dynamics in atomic junctions with two bosonic species, J. Phys. B: At. Mol. Opt. Phys. 44, 035301 (2011).

[21] J. Polo, A. Benseny, Th. Busch, V. Ahufinger, and J. Mompart, Transport of ultracold atoms between concentric traps via spatial adiabatic passage, New J. Phys. 18, 015010 (2016).

[22] C. Paré and M. Fłorjańczyk, Approximate model of soliton dynamics in all-optical couplers, Phys. Rev. A 41, 6287 (1990).

[23] A. I. Maimistov, Propagation of a light pulse in nonlinear tunnel-coupled optical waveguides, Kvant. Elektron. 18, 758 (1991) [Sov. J. Quantum Electron. 21, 687 (1991)].

[24] I. M. Uzunov, R. Muschall, M. Goelles, Yu. S. Kivshar, B. A. Malomed, and F. Lederer, Pulse switching in nonlinear fiber directional couplers, Phys. Rev. E 51, 2527 (1995).

[25] I. Bugar, I. V. Fedotov, A. B. Fedotov, M. Koys, R Buczynski, D. Pysz, J. Chlpik, F. Uherek, and A. M. Zheltikov, Polarization-controlled dispersive wave redirection in dual-core photonic crystal fiber, Laser Phys. 18, 1420 (2008).

[26] M. Abbarchi, A. Amo, V. G. Sala, D. D. Solnyshkov, H. Flayac, L. Ferrier, I. Sagnes, E. Galopin, A. Lemaître, G. Malpuech, and J. Bloch, Macroscopic quantum self-trapping and Josephson oscillations of exciton polaritons, Nat. Phys. 9, 275 (2013).

[27] J. E. Mooij, T. P. Orlando, L. Levitov, L. Tian, C. H. van der Wal, and S. Lloyd, Josephson persistent-current qubit, Science 285, 1036 (1999).

[28] Y. Makhlin, G. Schön, and A. Shnirman, Quantum-state engineering with Josephson-junction devices, Rev. Mod. Phys. 73, 357 (2001).

[29] I. Lesanovsky and W. von Klitzing, Spontaneous Emergence of Angular Momentum Josephson Oscillations in Coupled Annular Bose-Einstein Condensates, Phys. Rev. Lett. 98, 050401 (2007).

[30] J. Brand, T. J. Haigh, and U. Zülicke, Sign of coupling in barrier-separated Bose-Einstein condensates and 
stability of double-ring systems, Phys. Rev. A 81, 025602 (2010).

[31] D. Aghamalyan, L. Amico, and L. C. Kwek, Effective dynamics of cold atoms flowing in two ring-shaped optical potentials with tunable tunneling, Phys. Rev. A 88, 063627 (2013).

[32] S. W. Su, S. C. Gou, A. Bradley, O. Fialko, and J. Brand, Kibble-Zurek Scaling and its Breakdown for Spontaneous Generation of Josephson Vortices in Bose-Einstein Condensates, Phys. Rev. Lett. 110, 215302 (2013).

[33] J. Polo, J. Mompart, and V. Ahufinger, Geometrically induced complex tunnelings for ultracold atoms carrying orbital angular momentum, Phys. Rev. A 93, 033613 (2016).

[34] Z. Chen, Y. Li, B. A. Malomed, and L. Salasnich, Spontaneous symmetry breaking of fundamental states, vortices, and dipoles in two and one-dimensional linearly coupled traps with cubic self-attraction, Phys. Rev. A 96, 033621 (2016).

[35] A. Oliinyk, A. Yakimenko, and B. Malomed, Tunneling of persistent currents in coupled ring-shaped Bose-Einstein condensates, J. Phys. B: At. Mol. Opt. Phys. 52, 225301 (2019).

[36] A. Oliinyk, I. Yatsuta, B. Malomed, and A. Yakimenko, Symmetry breaking in interacting ring-shaped superflows of BoseEinstein condensates, Symmetry 11, 1312 (2019).

[37] A. Escrivà, A. Muñoz Mateo, M. Guilleumas, and B. Juliá-Díaz, Tunneling vortex dynamics in linearly coupled Bose-Hubbard rings, Phys. Rev. A 100, 063621 (2019).

[38] A. Oliinyk, B. Malomed, and A. Yakimenko, Nonlinear dynamics of Josephson vortices in merging superfluid rings, Comm. Nonlin. Sci. Num. Sim. 83, 105113 (2020).

[39] D. V. Averin, Coulomb Blockade in Superconducting Quantum Point Contacts, Phys. Rev. Lett. 82, 3685 (1999).

[40] H. Saito, Y. Kawaguchi, and M. Ueda, Breaking of Chiral Symmetry and Spontaneous Rotation in a Spinor Bose-Einstein Condensate, Phys. Rev. Lett. 96, 065302 (2006).

[41] M. Diez, I. C. Fulga, D. I. Pikulin, M. Wimmer, A. R. Akhmerov, and C. W. J. Beenakker, Phase-locked magnetoconductance oscillations as a probe of Majorana edge states, Phys. Rev. B 87, 125406 (2013).

[42] M. Liu, D. A. Powell, I. V. Shadrivov, M. Lapine, and Y. S. Kivshar, Spontaneous chiral symmetry breaking in metamaterials, Nat. Commun. 5, 4441 (2014)

[43] U. Khanna, D. K. Mukherjee, A. Kundu, and S. Rao, Chiral nodes and oscillations in the Josephson current in Weyl semimetals, Phys. Rev. B 93, 121409 (2016).

[44] C.-Z. Chen, J. J. He, D.-H. Xu, and K. T. Law, Emergent Josephson current of $N=1$ chiral topological superconductor in quantum anomalous Hall insulator/superconductor heterostructures, Phys. Rev. B 98, 165439 (2018).

[45] Y.-J. Lin, K. Jiménez-García, and I. B. Spielman, Spin-orbitcoupled Bose-Einstein condensates, Nature (London) 471, 83 (2011).

[46] V. Galitski and I. B. Spielman, Spin-orbit coupling in quantum gases, Nature (London) 494, 49 (2013).

[47] N. Goldman, G. Juzeliūnas, P. Öhberg, and I. B. Spielman, Light-induced gauge fields for ultracold atoms, Rep. Prog. Phys. 77, 126401 (2014).

[48] H. Zhai, Degenerate quantum gases with spin-orbit coupling: a review, Rep. Prog. Phys. 78, 026001 (2015).

[49] X.-F. Zhang, R.-F. Dong, T. Liu, W. M. Liu, and S.-G. Zhang, Spin-orbit-coupled Bose-Einstein condensates confined in concentrically coupled annular traps, Phys. Rev. A 86, 063628 (2012).

[50] Y. A. Bychkov and E. I. Rashba, Oscillatory effects and the magnetic susceptibility of carriers in inversion layers, J. Phys. C 17, 6039 (1984).

[51] D. S. Petrov, Quantum Mechanical Stabilization of a Collapsing Bose-Bose Mixture, Phys. Rev. Lett. 115, 155302 (2015).

[52] G. Fibich, The Nonlinear Schrödinger Equation: Singular Solutions and Optical Collapse (Springer, Heidelberg, 2015).

[53] H. Sakaguchi, B. Li, and B. A. Malomed, Creation of twodimensional composite solitons in spin-orbit-coupled selfattractive Bose-Einstein condensates in free space, Phys. Rev. E 89, 032920 (2014).

[54] B. Ramachandhran, B. Opanchuk, X.-J. Liu, H. Pu, P. D. Drummond, and $\mathrm{H}$. Hu, Half-quantum vortex state in a spinorbit-coupled Bose-Einstein condensate, Phys. Rev. A 85, 023606 (2012).

[55] M. F. Andersen, C. Ryu, P. Cladé, V. Natarajan, A. Vaziri, K. Helmerson, and W. D. Phillips, Quantized Rotation of Atoms from Photons with Orbital Angular Momentum, Phys. Rev. Lett. 97, 170406 (2006).

[56] R. Pugatch, M. Shuker, O. Firstenberg, A. Ron, and N. Davidson, Topological Stability of Stored Optical Vortices, Phys. Rev. Lett. 98, 203601 (2007).

[57] N. Lo Gullo, S. McEndoo, T. Busch, and M. Paternostro, Vortex entanglement in Bose-Einstein condensates coupled to Laguerre-Gauss beams, Phys. Rev. A 81, 053625 (2010).

[58] J. H. V. Nguyen, P. Dyke, D. Luo, B. A. Malomed, and R. G. Hulet, Collisions of matter-wave solitons, Nat. Phys. 10, 918 (2014).

[59] H. Sakaguchi, E. Ya. Sherman, and B. A. Malomed, Vortex solitons in two-dimensional spin-orbit coupled Bose-Einstein condensates: Effects of the Rashba-Dresselhaus coupling and the Zeeman splitting, Phys. Rev. E 94, 032202 (2016).

[60] G. Dresselhaus, Spin-Orbit coupling effects in zinc blende structures, Phys. Rev. 100, 580 (1955).

[61] S. V. Manakov, On the theory of two-dimensional stationary self-focusing of electromagnetic waves, Sov. Phys. JETP 38, 248 (1974) [Zh. Eksp. Teor. Fiz. 65, 505 (1973)].

[62] M. Rader, M. Hebenstreit, and R. E. Zillich, Multicomponent correlated-basis-function method and its application to multilayered dipolar Bose gases, Phys. Rev. A 95, 033625 (2017).

[63] A. Gubeskys and B. A. Malomed, Spontaneous soliton symmetry breaking in two-dimensional coupled Bose-Einstein condensates supported by optical lattices, Phys. Rev. A 76, 043623 (2007).

[64] J. Durnin, Exact solutions for nondiffractive beams. I. The scalar theory, J. Opt. Soc. Am. A 4, 651 (1987).

[65] D. McGloin and K. Dholakia, Bessel beams: diffraction in a new light, Contempr. Phys. 46, 15 (2005).

[66] A. Ortiz-Ambriz, S. Lopez-Aguayo, Y. V. Kartashov, V. A. Vysloukh, D. Petrov, H. Garcia-Gracia, J. C. Gutierrez-Vega, and L. Torner, Generation of arbitrary complex quasi-nondiffracting optical patterns, Opt. Express 21, 22221 (2013).

[67] R. Zhong, Z. Chen, C. Huang, Z. Luo, H. Tan, B. A. Malomed, and Y. Li, Self-trapping under the two-dimensional spin-orbitcoupling and spatially growing repulsive nonlinearity, Front. Phys. 13, 130311 (2018). 
[68] A. R. Champneys, B. A. Malomed, J. Yang, and D. J. Kaup, "Embedded solitons": Solitary waves in resonance with the linear spectrum, Physica D 152-153, 340 (2001).

[69] J. Yang, Nonlinear Waves in Integrable and Nonintegrable Systems (SIAM, 2010).

[70] H. Sakaguchi, B. Li, E. Ya. Sherman, and B. A. Malomed, Composite solitons in two-dimensional spin-orbit coupled selfattractive Bose-Einstein condensates in free space, Rom. Rep. Phys. 70, 502 (2018).

[71] D. S. Petrov and G. E. Astrakharchik, Ultradilute LowDimensional Liquids, Phys. Rev. Lett. 117, 100401 (2016).

[72] I. Ferrier-Barbut, H. Kadau, M. Schmitt, M. Wenzel, and T. Pfau, Observation of Quantum Droplets in a Strongly Dipolar Bose Gas, Phys. Rev. Lett. 116, 215301 (2016).

[73] L. Chomaz, S. Baier, D. Petter, M. J. Mark, F. Wähtler, L. Santos, F. Ferlaino, Quantum-Fluctuation-Driven Crossover from a Dilute Bose-Einstein Condensate to a Macrodroplet in a Dipolar Quantum Fluid, Phys. Rev. X 6, 041039 (2016).

[74] C. R. Cabrera, L. Tanzi, J. Sanz, B. Naylor, P. Thomas, P. Cheiney, and L. Tarruell, Quantum liquid droplets in a mixture of Bose-Einstein condensates, Science 359, 301 (2018).
[75] G. Semeghini, G. Ferioli, L. Masi, C. Mazzinghi, L. Wolswijk, F. Minardi, M. Modugno, G. Modugno, M. Inguscio, and M. Fattori, Self-Bound Quantum Droplets in Atomic Mixtures, Phys. Rev. Lett. 120, 235301 (2018).

[76] C. D’Errico, A. Burchiant, M. Prevedelli, L. Salasnich, F. Ancilotto, M. Modugno, F. Minardi, and C. Fort, Observation of quantum droplets in a heteronuclear bosonic mixture, Phys. Rev. Research 1, 033155 (2019).

[77] Y. Li, Z. Luo, Y. Liu, Z. Chen, C. Huang, S. Fu, H. Tan, and B. A. Malomed, Two-dimensional solitons and quantum droplets supported by competing self- and cross-interactions in spin-orbit-coupled condensates, New J. Phys. 19, 113043 (2017).

[78] Y. V. Kartashov, B. A. Malomed, V. V. Konotop, V. E. Lobanov, and L. Torner, Stabilization of spatiotemporal solitons in Kerr media by dispersive coupling, Opt. Lett. 40, 1045 (2015).

[79] E. Shamriz, Z. Chen, and B. A. Malomed, Stabilization of onedimensional Townes solitons by spin-orbit coupling in a dualcore system, Comm. Nonlin. Sci. Numer. Simul. 91, 105412 (2020). 